\title{
Blood Agar Growth Medium
}

National Cancer Institute

\section{Source}

National Cancer Institute. Blood Agar Growth Medium. NCI Thesaurus. Code C127517.

A solid and selective growth medium containing agar and blood; it allows for the

cultivation and differentiation of microorg anisms based on hemolytic activity. 